\title{
Small Alcohols Destabilize the KcsA Tetramer via Their Effect on the Membrane Lateral Pressure ${ }^{\dagger}$
}

\author{
Els van den Brink-van der Laan,* Vladimir Chupin, J. Antoinette Killian, and Ben de Kruijff \\ Department of Biochemistry of Membranes, Centre for Biomembranes and Lipid Enzymology, Institute of Biomembranes, \\ Utrecht University, Padualaan 8, 3584 CH Utrecht, The Netherlands
}

Received February 26, 2004; Revised Manuscript Received April 7, 2004

\begin{abstract}
Previously, it was shown that the tetrameric potassium channel KcsA when present in a lipid bilayer can be dissociated by trifluoroethanol [van den Brink-van der Laan, E., et al. (2004) Biochemistry $43,4240-4250]$. Here, we demonstrate that this is a general property of small alcohols. We found that small alcohols dissociate the KcsA tetramer, at a concentration that depends on their membrane affinity. Importantly, the efficiency of the alcohol-induced tetramer dissociation was found to correlate with the efficiency of both alcohol-induced bilayer leakage and acyl chain disordering. Our data suggest that the ability of small alcohols to induce KcsA tetramer dissociation and to function as anesthetics depends on their effect on the membrane lateral pressure.
\end{abstract}

Many membrane proteins function as oligomeric complexes. The formation of these complexes is essential for their function. An example is the tetrameric membrane protein KcsA from Streptomyces lividans, in which the four subunits together function as a potassium channel. KcsA is often used as model protein for ion channels and oligomeric membrane proteins $(1-8)$, since the protein is wellcharacterized in biochemical and electrophysiological experiments $(9-11)$ and the structure of the protein is known (12, 13). The four subunits of KcsA are arranged symmetrically around the central pore. Each subunit consists of an Nterminal helix lying at the membrane interface, two transmembrane helices separated by a pore region, and a large C-terminal cytoplasmic domain.

The tetrameric structure of KcsA is highly stable in a wide range of detergents, even in SDS (10). This high stability is caused not only by interactions between protein subunits but also by interactions between the protein and the surrounding lipid bilayer. For example, it has been shown that the efficiency of formation of the the KcsA tetramer during assembly depends on the membrane lipid composition (6). Also, the thermostability of KcsA is dependent on the nature of the lipid environment (6).

Both specific and general lipid-protein interactions can play a role in the stabilization of membrane protein oligomers. A unifying principle for general protein-lipid interactions is the membrane lateral pressure. This lateral pressure varies with the depth within the bilayer $(14,15)$. A large negative lateral pressure is localized at the interface between the headgroups and acyl chain region, due to the interfacial tension. This is compensated by a positive lateral pressure in the headgroup region due to repulsions between the headgroups and a positive lateral pressure in the acyl chain

$\dagger$ This work was supported by the Dutch Foundation for Fundamental Research on Matter (FOM).

* To whom correspondence should be addressed. Fax: (31)(30)2533969. Telephone: (31)(30)2532465. E-mail: e.vandenbrink@ chem.uu.nl. region due to acyl chain repulsions $(14,15)$. The resulting lateral pressure profile is sensitive to changes in the lipid composition (16) and the presence of small solutes (17). Changes in the lateral pressure have been suggested to modulate protein function (14), e.g., for the bacterial translocase secYEG (18) and the ion channel-forming peptide alamethicin $(19,20)$. For this peptide, it was also calculated that changes in the lateral pressure profile affect the size distribution of the peptide oligomers, in qualitative agreement with experimental results (20).

Recently, the unexpected observation that 2,2,2-trifluoroethanol (TFE) $)^{1}$ can induce dissociation of the KcsA tetramer was made $(21-23)$. We showed that this was caused not only by direct interactions between TFE and KcsA but also predominantly by an indirect effect via changes in the stabilizing general interactions between KcsA and the surrounding lipids (23). A model was proposed in which TFE inserts in the headgroup region of the lipid bilayer, resulting in an increased lateral pressure in the headgroup region, compensated by a lower lateral pressure in the acyl chain region. The latter results in destabilization of the KcsA tetramer (23).

In this paper, we investigated if the observed effects are specific for TFE, or if they are a general effect of small alcohols, related to their membrane affinity. Experiments were carried out to determine the effect of small alcohols on KcsA tetramer stability and on the lipid bilayer structure. The obtained data imply that small alcohols destabilize KcsA

${ }^{1}$ Abbreviations: BuOH, 1-butanol; CF, carboxyfluorescein; DDM, $n$-dodecyl $\beta$-D-maltoside; DOPC, 1,2-dioleoyl-sn-glycero-3-phosphocholine; $\Delta v_{\mathrm{q}}$, quadrupolar splitting; EtOH, ethanol; $\left[{ }^{2} \mathrm{H}_{4}\right] \mathrm{DOPC}$, DOPC labeled with two deuterium atoms on both acyl chains at the $\mathrm{C} 11$ position; HFIP, 1,1,1,3,3,3-hexafluoro-2-propanol; $\mathrm{H}_{\text {II }}$, hexagonal phase; IP, 2-propanol; $\mathrm{L}_{\alpha}$, liquid crystalline phase; LUVs, large unilamellar vesicles prepared by extrusion techniques; $\mathrm{MeOH}$, methanol; NMR, nuclear magnetic resonance; PC, phosphatidylcholine; $P_{\text {ow }}$, octanolwater partitioning coefficient; ProOH, 1-propanol; SDS-PAGE, sodium dodecyl sulfate-polyacrylamide gel electrophoresis; TFE, 2,2,2trifluoroethanol. 
via changes in the membrane lateral pressure and suggest that also at lower concentrations, at which these alcohols can act as anesthetics, they interact with membrane proteins via changes in the lateral pressure profile.

\section{EXPERIMENTAL PROCEDURES}

Methanol (>99.9\%) and 1-butanol (>99.8\%) were from Sigma-Aldrich. Ethanol (>99.9\%) was obtained from Baker. 1-Propanol (>99\%), 2-propanol (>99.7\%), and 2,2,2-trifluoroethanol ( $>99 \%$ ) were purchased from Merck. 1,1,1,3,3,3Hexafluoro-2-propanol (>99.5\%) was obtained from Acros. All experiments were performed essentially as described previously (23), except that other alcohols were used instead of TFE. Briefly, KcsA was purified and reconstituted in DOPC vesicles at a protein (monomer):lipid molar ratio of $1: 1000$ and at a protein concentration of $\pm 0.1 \mathrm{mg} / \mathrm{mL}$. Alcohol-induced dissociation of the KcsA tetramer was studied by SDS-PAGE after incubation for $1 \mathrm{~h}$ and subsequent acetone precipitation to remove alcohol and lipids. The extent of membrane leakage was measured by fluorescence, using carboxyfluorescein (CF)-loaded vesicles (10 mM phospholipids). Twenty microliters of the vesicle suspension was mixed with the desired volume of alcohol and subsequently diluted into $1 \mathrm{~mL}$ of buffer, after which the fluorescence was measured immediately. ${ }^{2} \mathrm{H}$ NMR spectra were recorded at $76.8 \mathrm{MHz}$ for samples containing $2 \mu \mathrm{mol}$ of DOPC, labeled with two deuterium atoms on both acyl chains at the $\mathrm{C} 11$ position. Samples were hydrated with 100 $\mu \mathrm{L}$ of deuterium-depleted buffer in the absence or presence of $0.2 \mathrm{M}$ alcohol. Samples containing $0.6 \mathrm{M}$ alcohol were prepared by addition of alcohol to the samples containing $0.2 \mathrm{M}$ alcohol, followed by two freeze-thaw cycles.

\section{RESULTS}

Alcohol-Induced Tetramer Dissociation. Dissociation of the potassium channel KcsA into the monomeric form induced by TFE can be followed by SDS-PAGE (23). Therefore, this method was used to compare the effect of TFE with that of other alcohols that can partition into lipid bilayers. As an example, Figure 1A shows an SDS-PAGE gel of KcsA, reconstituted in DOPC and incubated with methanol $(\mathrm{MeOH})$, ethanol (EtOH), or propanol (ProOH) at two different concentrations. It can be seen in this figure that all three alcohols induce tetramer dissociation, resulting mainly in monomeric KcsA, although also some KcsA aggregates are formed, which run high in the gel. As can also be seen in Figure 1A, the concentration of the alcohols required to induce KcsA tetramer dissociation is different for the three alcohols and the efficiency increases going from $\mathrm{MeOH}$ to $\mathrm{EtOH}$ and $\mathrm{ProOH}$. Similar experiments were performed with these and other alcohols over a large concentration range. The quantified results are shown in Figure 1B. Butanol $(\mathrm{BuOH})$, which could be tested only at concentrations below the solubility limit of $0.8 \mathrm{M}$, is not included in this figure, since we observed no effect of this alcohol in that concentration range. All other tested alcohols are fully miscible with water and induced KcsA tetramer dissociation, but at very different concentrations, ranging from $200 \mathrm{mM}$ for 1,1,1,3,3,3-hexafluoro-2-propanol (HFIP) to produce $50 \%$ KcsA tetramer dissociation to $18 \mathrm{M}$ for $\mathrm{MeOH}$. Interestingly, the efficiency of the alcohols in
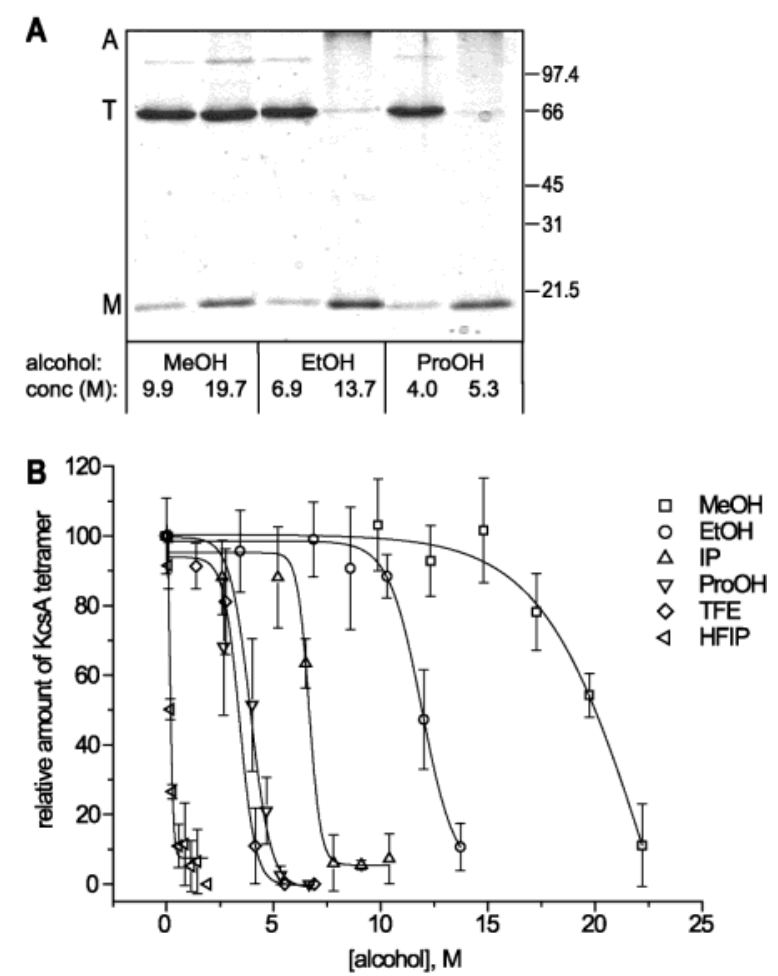

FIGURE 1: Alcohol-induced dissociation of the KcsA tetramer. $\mathrm{KcsA}$, reconstituted in DOPC at a protein (monomer):lipid molar ratio of 1:1000, was incubated with various concentrations of the different alcohols for $1 \mathrm{~h}$ at room temperature. Samples were analyzed by SDS-PAGE after acetone precipitation. Coomassiestained gels were quantified by densitometry. (A) SDS-PAGE of $\mathrm{Kcs}$, incubated with methanol $(\mathrm{MeOH})$, ethanol $(\mathrm{EtOH})$, or propanol $(\mathrm{ProOH})$ at different concentrations. Monomeric (M), tetrameric (T), and aggregated (A) KcsA are denoted, and a protein size marker (in kilodaltons) is shown at the right. (B) Relative amount of KcsA tetramer (relative to the sample without any alcohol, analyzed on the same SDS-PAGE gel), incubated with different alcohols at various concentrations, as determined by SDSPAGE. The standard deviation is given, based on at least three different experiments. Data points were fit by a sigmoidal fit.

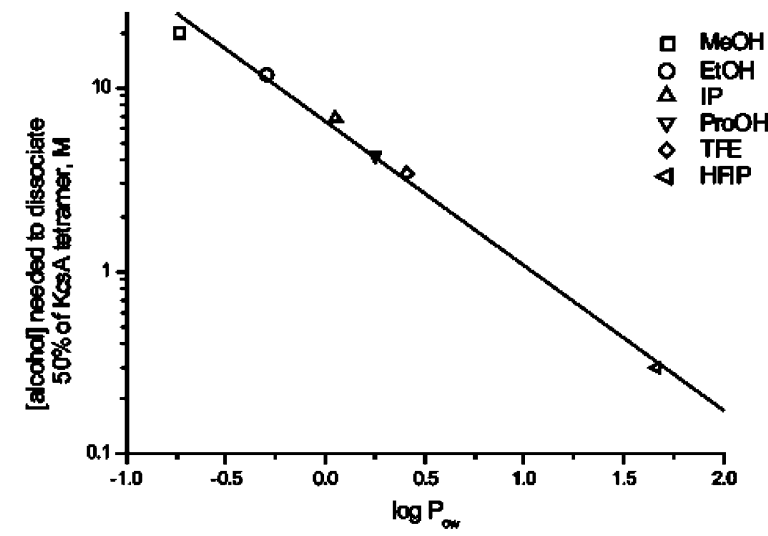

FIGURE 2: Correlation between the alcohol concentration needed for KcsA tetramer dissociation and the octanol-water partitioning coefficient $\left(\log P_{\mathrm{ow}}\right)$. Octanol-water partitioning coefficients were obtained from ref 42 . The data were fit exponentially (note the logarithmic $y$-axis) with a correlation coefficient of 0.99 .

inducing KcsA tetramer dissociation correlates with their octanol-water partitioning coefficients, as is shown in Figure 2 , and hence with their ability to partition into the lipid bilayer (24). This correlation suggests that the alcohols induce dissociation of the KcsA tetramer indirectly via their effect 

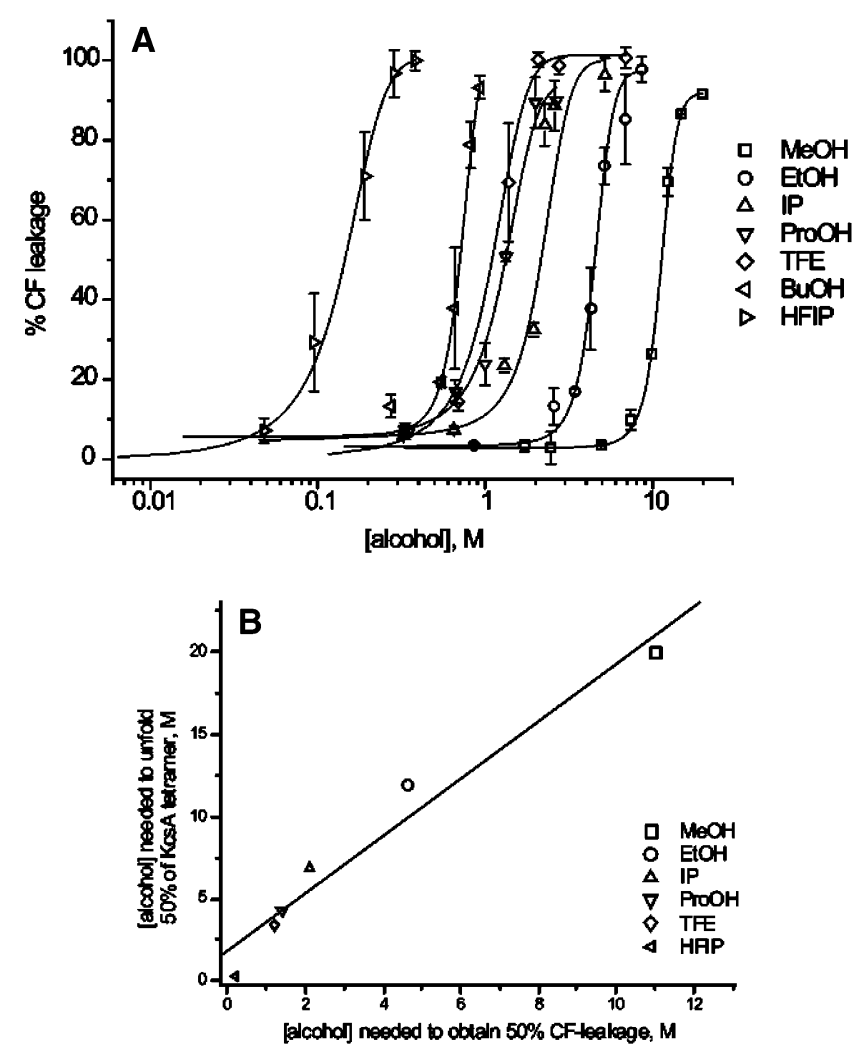

FIGURE 3: Carboxyfluorescein leakage of DOPC vesicles, induced by alcohol. (A) Various concentrations of the different alcohols were added to carboxyfluorescein-loaded vesicles. The extent of leakage was determined immediately after dilution with buffer in the cuvette. The standard deviation is based on at least three different measurements. Data points were fitted by a sigmoidal fit. Note the logarithmic $x$-axis. (B) Correlation between KcsA tetramer stability and the carboxyfluorescein leakage. The concentration of alcohol needed to dissociate $50 \%$ of the KcsA tetramer (derived from Figure 1) is plotted against the concentration of alcohol needed to induce $50 \%$ CF leakage (derived from Figure 2).

on the lipids, similar to what we proposed previously for the interaction between KcsA and TFE (23).

Effect of Alcohols on Lipid Bilayer Organization. Next, we investigated the effect of small alcohols on the barrier properties of the lipid bilayer. Therefore, carboxyfluorescein leakage experiments were performed. Figure $3 \mathrm{~A}$ shows that all alcohols induce bilayer leakage. Although the concentrations needed to induce bilayer leakage are lower than those required for dissociation of the KcsA tetramer, the order of efficiency between the alcohols is the same. The alcohol concentration needed for tetramer dissociation increases with the alcohol concentration required for bilayer leakage in a nearly linear relationship with a correlation coefficient of 0.96, as shown in Figure 3B. This supports our suggestion that the alcohols interact indirectly with KcsA via the surrounding lipids.

To obtain a better understanding of the effect of the alcohols on the lipid bilayer, we studied their effect on the acyl chain ordering. For this, ${ }^{2} \mathrm{H}$ NMR was used for samples containing DOPC, deuterated at the 11 position of the oleoyl chains. The quadrupolar splitting $\left(\Delta v_{\mathrm{q}}\right)$, measured as the distance between the two peaks in the spectra, is directly related to the acyl chain order, with decreasing chain order resulting in smaller values of $\Delta v_{\mathrm{q}} \cdot{ }^{2} \mathrm{H}$ NMR spectra were recorded for samples containing $0.2 \mathrm{M}$ alcohol, which, except for the case of HFIP, is below the concentration needed to induce KcsA tetramer dissociation or bilayer leakage. Figure $4 \mathrm{~A}$ shows that the presence of this low concentration of the alcohols already significantly decreases the quadrupolar splitting. The order of efficiency of the different alcohols in lowering $\Delta v_{\mathrm{q}}$ is similar to what was found for KcsA tetramer dissociation and carboxyfluorescein leakage. HFIP was not included in this figure, because the presence of 0.2 M HFIP already disrupted the bilayer organization (data not shown). However, at a concentration of $0.02 \mathrm{M}$, we found that the bilayer structure was still intact, and $\Delta v_{\mathrm{q}}$ was decreased from 6.2 to $4.7 \mathrm{kHz}$ (data not shown), which is comparable to the effect measured for $0.2 \mathrm{M}$ TFE. Increasing the concentration of the other alcohols to $0.6 \mathrm{M}$ resulted in a further decrease in the quadrupolar splitting, as indicated in Figure 4B. Also, at this higher alcohol concentration, the order of efficiency of the different alcohols is similar to what was found before for KcsA tetramer dissociation and carboxyfluorescein leakage (see above), demonstrating that dissociation of the KcsA tetramer is related to a decrease in the extent of acyl chain packing.

\section{DISCUSSION}

In this study, we have investigated the effect of various alcohols on the stability of the KcsA tetramer and on the surrounding DOPC bilayer. Previously, it was found that TFE can induce dissociation of the KcsA tetramer via changes in the membrane lateral pressure (23). Here, we show that also other alcohols can induce KcsA tetramer dissociation and that this occurs via a similar mechanism, depending on their membrane affinity.

The octanol-water partitioning coefficient is a hydrophobicity measure which indicates the membrane affinity of such molecules (24). The observed correlation between the ability of the alcohols to induce KcsA tetramer dissociation and their octanol-water partitioning coefficients indicates that these alcohols insert into the lipid bilayer, and interact indirectly with the KcsA tetramer via the surrounding lipids. This is supported by the observed correlation between the alcoholinduced KcsA tetramer dissociation and bilayer leakage.

Previously, we have shown by NMR that when TFE inserts into the lipid bilayer, this molecule localizes preferentially in the lipid headgroup region of the membrane (23). A similar localization was also described for ethanol $(25,26)$. It is likely that the other alcohols that we investigated here also partition mainly in the lipid headgroup region. The observed effects of these alcohols on the acyl chain packing support such localization.

Changes in the acyl chain order, as indicated by the ${ }^{2} \mathrm{H}$ NMR measurements, are related to changes in the membrane lateral pressure in the acyl chain region (27). The membrane lateral pressure depends on the interactions between membrane constituents and varies with the depth within the bilayer $(14,28)$. The lateral pressure is positive in the headgroup and acyl chain region due to repulsions between lipid headgroups and acyl chains, respectively, which are compensated by a negative lateral pressure at the interface between the lipid headgroups and acyl chains due to surface tension. As a result of the partitioning of the alcohols in the lipid headgroup region, we suggest that a redistribution of lateral pressures takes place from the interior of the bilayer toward the aqueous interfacial regions. 

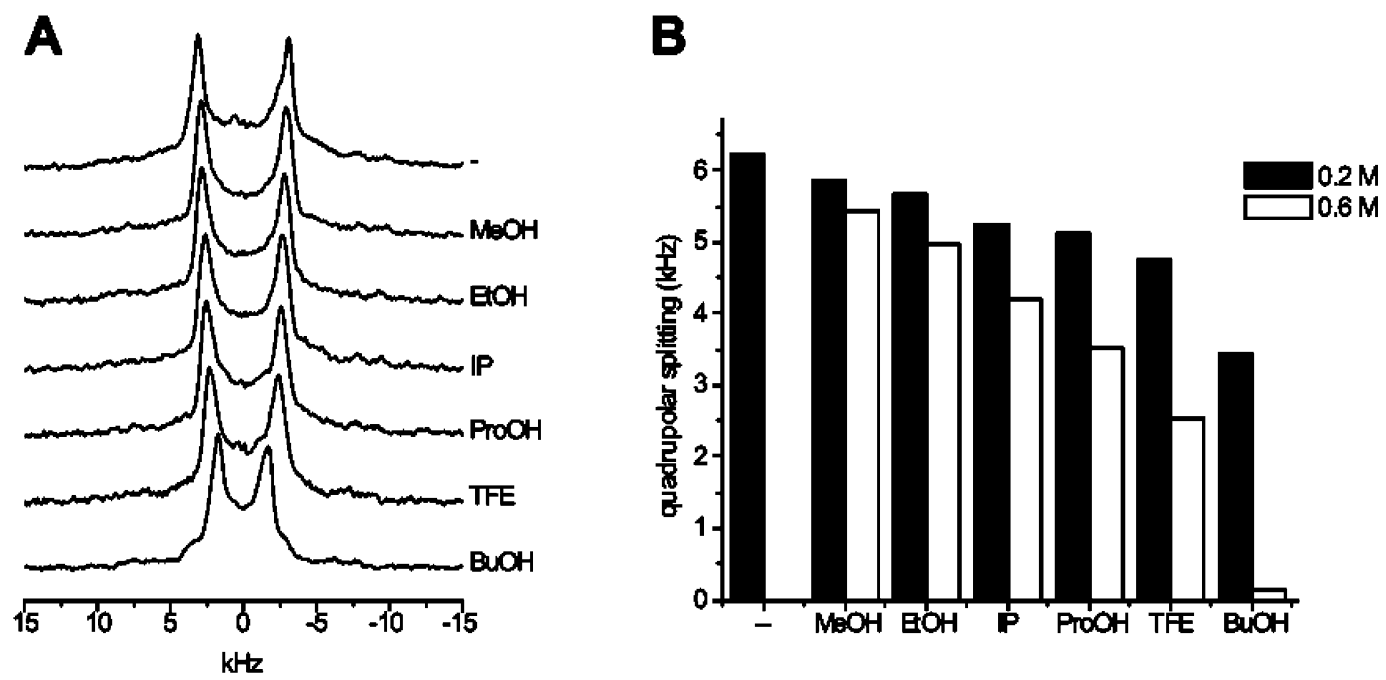

FIGURE 4: ${ }^{2} \mathrm{H}$ NMR data of $\left[{ }^{2} \mathrm{H}_{4}\right] \mathrm{DOPC}$ in the presence of the different alcohols at 0.2 and $0.6 \mathrm{M} .{ }^{2} \mathrm{H}$ NMR spectra were recorded for 2 $\mu \mathrm{mol}$ of $\left[{ }^{2} \mathrm{H}_{4}\right]$ DOPC in $100 \mu \mathrm{L}$ of buffer containing the different alcohols at 0.2 or $0.6 \mathrm{M}$. Spectra were recorded at room temperature. (A) ${ }^{2} \mathrm{H}$ NMR spectra of samples containing $0.2 \mathrm{M}$ alcohol. (B) Quadrupolar splittings of ${ }^{2} \mathrm{H}$ NMR spectra of $\left[{ }^{2} \mathrm{H}_{4}\right] \mathrm{DOPC}$ in the presence of the different alcohols at 0.2 or $0.6 \mathrm{M}$. The left-most bar represents the quadrupolar splitting measured for a control sample without alcohol.

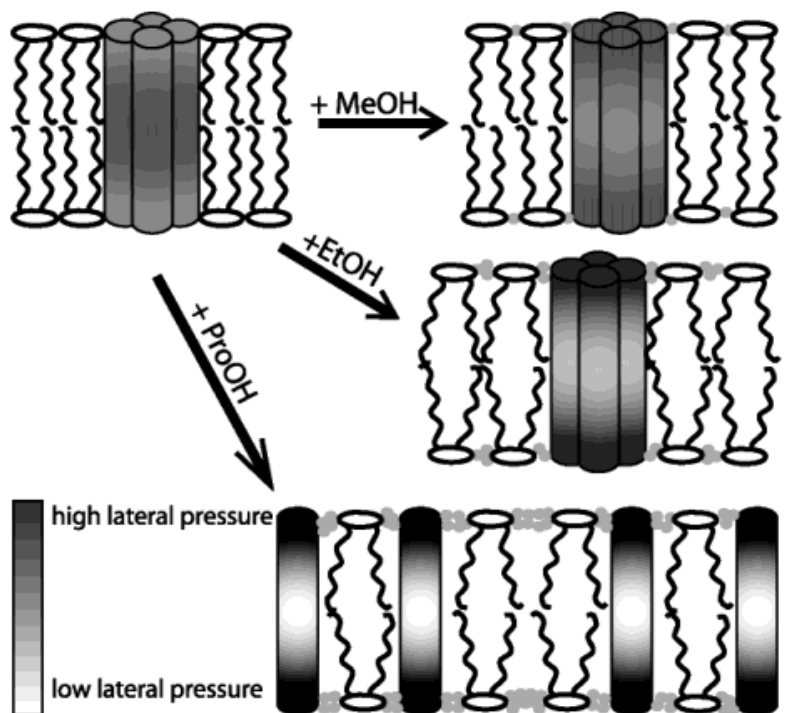

FIGURE 5: Model of the interaction between the alcohols and the KcsA tetramer via the membrane lateral pressure. For simplicity, a KcsA monomer is represented as a cylinder. The coloring of the cylinders indicates the positive lateral pressure acting on the KcsA tetramer, with dark coloring representing a high positive lateral pressure and light coloring representing a low positive lateral pressure. For simplicity, the negative lateral pressure at the interface between the headgroups and acyl chains is not indicated in the model. The effect of the addition of $\pm 7 \mathrm{M} \mathrm{MeOH}, \mathrm{EtOH}$, or ProOH on the lateral pressure and the KcsA tetramer dissociation is indicated. As a result of the different octanol-water partitioning coefficients of $\mathrm{MeOH}, \mathrm{EtOH}$, and $\mathrm{ProOH}$, the number of alcohol molecules that partition into the membrane is different for these alcohols, resulting in a different effect on the lateral pressure. This model is an extension of what we have shown previously for the effect of TFE on the KcsA tetramer in a lipid bilayer (23).

Figure 5 shows a semiquantitative model that describes the mechanism via which small alcohols destabilize an oligomeric membrane protein such as KcsA. The membrane affinity is increased upon going from $\mathrm{MeOH}$ to ProOH, resulting in a larger number of alcohol molecules that partition into the lipid bilayer. As a result of this partitioning, the lateral pressure profile in the membrane is changed. The lower lateral pressure in the acyl chain region destabilizes
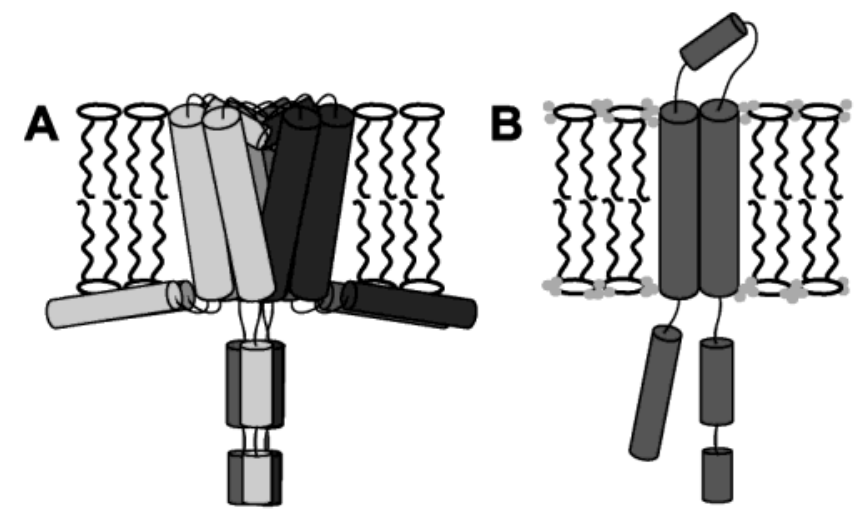

FIGURE 6: Model for the structure of the KcsA tetramer (A) and the KcsA monomer after the alcohol-induced tetramer dissociation (B). See the text for further comments.

the KcsA tetramer and finally induces tetramer dissociation, as indicated in the model for ProOH.

Changes in the membrane lateral pressure will affect only membrane proteins whose function depends on a transition between conformational states that is accompanied by a depth-dependent change in the cross-sectional area $(14,29)$. For example, it has been suggested that an increased acyl chain lateral pressure will stimulate a transition from a cylindrically shaped membrane protein structure to an hourglass-shaped membrane protein structure $(14,29)$. Therefore, small alcohols can only induce KcsA tetramer dissociation via their effect on the membrane lateral pressure, if this dissociation is accompanied by a change in the shape of the KcsA protein molecules. The crystal structure of KcsA shows that the transmembrane domain of the KcsA tetramer has an inverted conical (also called inverted teepee) shape (12), as shown schematically in Figure 6A. However, the $\mathrm{N}$-terminal domain of the protein is an amphipathic helix that seems to be inserted in the membrane interface (13). As a result, the narrowest part of the protein is probably located in the lower part of the transmembrane domain, just below the center of the lipid bilayer. The structure of the KcsA monomer is unknown, but it was shown recently that the pore region of monomeric KcsA is exposed outside the 

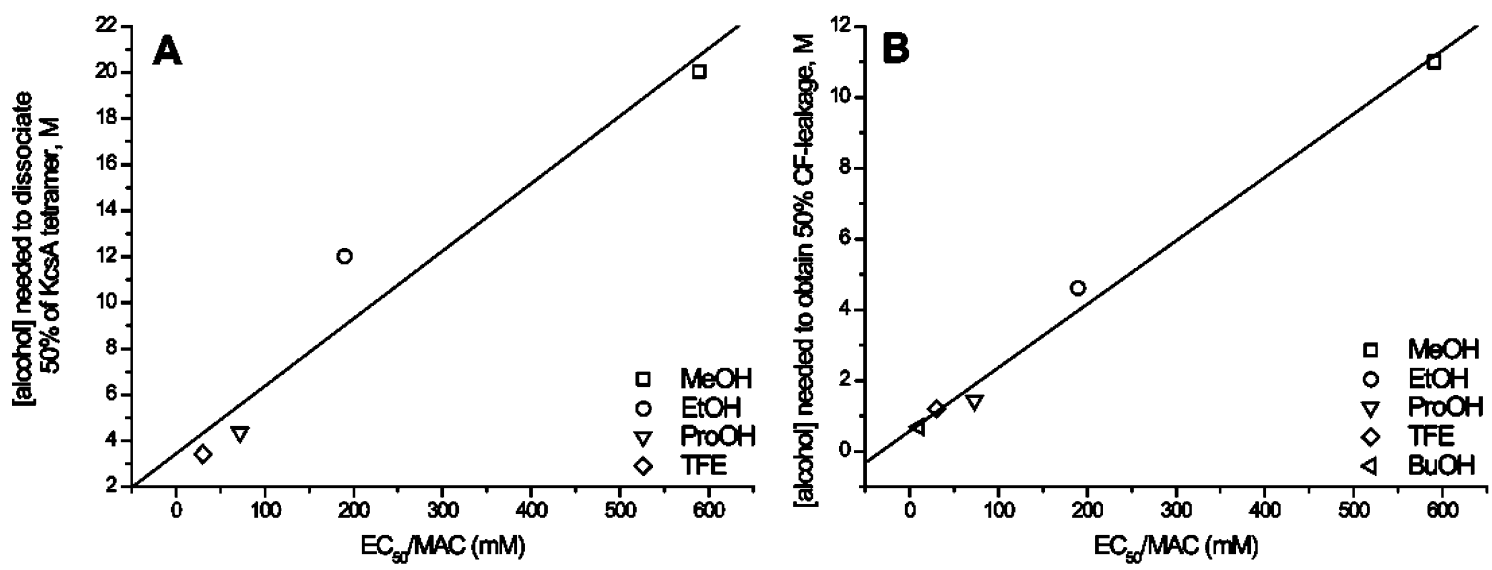

FIGURE 7: Correlation between the alcohol-induced KcsA tetramer dissociation, the carboxyfluorescein leakage, and the anesthetic potency of the different alcohols. The concentration of alcohol needed to dissociate $50 \%$ of the KcsA tetramer (A) and the concentration of alcohol needed to induce $50 \% \mathrm{CF}$-leakage (B) are plotted against the anesthetic potency, which is generally expressed as the $\mathrm{EC}_{50}($ for $\mathrm{MeOH}$ $\mathrm{EtOH}, \mathrm{ProOH}$, and $\mathrm{BuOH}$ ) or the minimum alveolar concentration (MAC, for TFE) (43). The best linear fit is shown. Data for IP and HFIP were not included in this figure, since the anesthetic potencies of this compounds were not available.

membrane during the topological membrane insertion $(8)$. We suggest that also during the disassembly of the KcsA tetramer, induced by the small alcohols, the pore domain of the monomeric KcsA becomes exposed, as shown schematically in Figure 6B. Furthermore, we suggest that after dissociation of the KcsA tetramer induced by the alcohols, the $\mathrm{N}$-terminal domain of the protein is not inserted into the lipid bilayer anymore, because of the partitioning of the alcohol in the lipid bilayer and the direct interactions of the alcohol molecules with the protein (see Figure 6B). The structure of the transmembrane helices in the monomer is not known, but most likely, the overall shape is more cylindrical than the KcsA subunit in the tetramer, due to the flipped-out pore region and $\mathrm{N}$-terminal domain. On this basis, we suggest that alcohol-induced dissociation of KcsA is accompanied by a transition from a (deformed) hourglassshaped structure to a more cylindrically shaped structure. Such a transition would be stimulated by a decrease in the acyl chain lateral pressure, caused by the membrane partitioning of alcohols, which is consistent with our results.

Most of the tested alcohols are general anesthetics. General anesthetics have been shown to affect the function of many membrane proteins, mainly receptors and ion channels (30, 31 ), including potassium channels (31-33). Meyer (34) and Overton (35) suggested for anesthetics an indirect interaction with these membrane proteins via the lipid bilayer, based on the correlation between the anesthetic potency and the octanol partitioning coefficient (36), similar to that is described here for KcsA tetramer dissociation. Interestingly, there is a linear relationship $\left(R^{2}=0.93\right)$ between the KcsA tetramer dissociation and the anesthetic potency (Figure 7A). Also, for the bilayer leakage (Figure 7B), a linear relationship $\left(R^{2}=0.99\right)$ with the anesthetic potency of the alcohols was found. These correlations suggest that a similar mechanism is involved in the KcsA tetramer dissociation and in anesthesia. However, this dissociation occurs at concentrations $(0.2-18 \mathrm{M})$ much higher than the concentrations needed for anesthesia (20-600 mM). Moreover, the alcoholinduced KcsA tetramer dissociation is most likely irreversible, as we showed previously for TFE (23), whereas the effect of anesthesia is reversible. Therefore, we suggest that the alcohols do not induce anesthesia via dissociation of oligomeric ion channels, but probably via more subtle changes in the protein structure, which could change their gating mechanism $(31-33)$. On one hand, it is possible that such protein structure changes are caused by direct interactions between anesthetics and hydrophobic sites of membrane proteins. Such a direct mechanism has been predicted, for example, because of exceptions to the Meyer-Overton correlation (37). On the other hand, it has been calculated by Cantor $(38,39)$ that general anesthetics shift the distribution of lateral pressure within the lipid bilayer, thereby inducing conformational changes in membrane proteins. Such an indirect mechanism via the lateral pressure profile could also explain many of the anomalies of the Meyer-Overton correlation (38-41). Cantor observed a qualitatively good agreement between the calculated change in the lateral pressure profile and the anesthetic potency $(38,39)$. In the study presented here, we observed a correlation between the alcohol-induced KcsA tetramer dissociation, the bilayer leakage, and the anesthetic potency of the alcohols. Together, these studies suggest that at both low and high concentrations the alcohols interact with membrane proteins via changes in the membrane lateral pressure.

\section{REFERENCES}

1. Irizarry, S. N., Kutluay, E., Drews, G., Hart, S. J., and Heginbotham, L. (2002) Opening the KcsA $\mathrm{K}^{+}$channel: tryptophan scanning and complementation analysis lead to mutants with altered gating, Biochemistry 41, 13653-13662.

2. Sansom, M. S., Shrivastava, I. H., Bright, J. N., Tate, J., Capener, C. E., and Biggin, P. C. (2002) Potassium channels: structures, models, simulations, Biochim. Biophys. Acta 1565, 294-307.

3. Kelly, B. L., and Gross, A. (2003) Potassium channel gating observed with site-directed mass tagging, Nat. Struct. Biol. 10, $280-284$

4. Nimigean, C. M., Chappie, J. S., and Miller, C. (2003) Electrostatic tuning of ion conductance in potassium channels, Biochemistry 42, 9263-9268.

5. van Dalen, A., Schrempf, H., Killian, J. A., and de Kruijff, B. (2000) Efficient membrane assembly of the KcsA potassium channel in Escherichia coli requires the protonmotive force, EMBO Rep. 1, 340-346.

6. van Dalen, A., Hegger, S., Killian, J. A., and de Kruijff, B. (2002) Influence of lipids on membrane assembly and stability of the potassium channel KcsA, FEBS Lett. 525, 33-38.

7. van Dalen, A., van der Laan, M., Driessen, A. J., Killian, J. A., and de Kruijff, B. (2002) Components required for membrane 
assembly of newly synthesized $\mathrm{K}^{+}$channel KcsA, FEBS Lett. 511, $51-58$.

8. Umigai, N., Sato, Y., Mizutani, A., Utsumi, T., Sakaguchi, M., and Uozumi, N. (2003) Topogenesis of two transmembrane type $\mathrm{K}^{+}$channels, Kir 2.1 and KcsA, J. Biol. Chem. 278, 4037340384.

9. Heginbotham, L., Odessey, E., and Miller, C. (1997) Tetrameric stoichiometry of a prokaryotic $\mathrm{K}^{+}$channel, Biochemistry 36, 10335-10342.

10. Cortes, D. M., and Perozo, E. (1997) Structural dynamics of the Streptomyces lividans $\mathrm{K}^{+}$channel (SKC1): oligomeric stoichiometry and stability, Biochemistry 36, 10343-10352.

11. Cuello, L. G., Romero, J. G., Cortes, D. M., and Perozo, E. (1998) $\mathrm{pH}$-dependent gating in the Streptomyces lividans $\mathrm{K}^{+}$channel, Biochemistry 37, 3229-3236.

12. Doyle, D. A., Morais Cabral, J., Pfuetzner, R. A., Kuo, A., Gulbis, J. M., Cohen, S. L., Chait, B. T., and MacKinnon, R. (1998) The structure of the potassium channel: molecular basis of $\mathrm{K}^{+}$ conduction and selectivity, Science 280, 69-77.

13. Cortes, D. M., Cuello, L. G., and Perozo, E. (2001) Molecular architecture of full-length KcsA: role of cytoplasmic domains in ion permeation and activation gating, J. Gen. Physiol. 117, 165180.

14. Cantor, R. S. (1997) Lateral pressures in cell membranes: a mechanism for modulation of protein function, J. Phys. Chem. B $101,1723-1725$.

15. Marsh, D. (1996) Components of the lateral pressure in lipid bilayers deduced from HII phase dimensions, Biochim. Biophys. Acta 1279, 119-123.

16. Cantor, R. S. (1999) Lipid composition and the lateral pressure profile in bilayers, Biophys. J. 76, 2625-2639.

17. Cantor, R. S. (1999) Solute modulation of conformational equilibria in intrinsic membrane proteins: apparent "cooperativity" without binding, Biophys. J. 77, 2643-2647.

18. van der Does, C., Swaving, J., van Klompenburg, W., and Driessen, A. J. (2000) Non-bilayer lipids stimulate the activity of the reconstituted bacterial protein translocase, J. Biol. Chem. 275, 2472-2478.

19. Keller, S. L., Bezrukov, S. M., Gruner, S. M., Tate, M. W., Vodyanoy, I., and Parsegian, V. A. (1993) Probability of alamethicin conductance states varies with nonlamellar tendency of bilayer phospholipids, Biophys. J. 65, 23-27.

20. Cantor, R. S. (2002) Size distribution of barrel-stave aggregates of membrane peptides: influence of the bilayer lateral pressure profile, Biophys. J. 82, 2520-2525.

21. Demmers, J. A., van Dalen, A., de Kruijff, B., Heck, A. J., and Killian, J. A. (2003) Interaction of the $\mathrm{K}^{+}$channel KcsA with membrane phospholipids as studied by ESI mass spectrometry, FEBS Lett. 541, 28-32.

22. Valiyaveetil, F. I., Zhou, Y., and MacKinnon, R. (2002) Lipids in the structure, folding, and function of the KcsA $\mathrm{K}^{+}$channel, Biochemistry 41, 10771-10777.

23. van den Brink-van der Laan, E., Chupin, V., Killian, J. A., and De Kruijff, B. (2004) Stability of KcsA tetramer depends on membrane lateral pressure, Biochemistry 43, 4240-4250.

24. Kepczynski, M., Pandian, R. P., Smith, K. M., and Ehrenberg, B. (2002) Do liposome-binding constants of porphyrins correlate with their measured and predicted partitioning between octanol and water? Photochem. Photobiol. 76, 127-134.
25. Holte, L. L., and Gawrisch, K. (1997) Determining ethanol distribution in phospholipid multilayers with MAS-NOESY spectra, Biochemistry 36, 4669-4674.

26. Feller, S. E., Brown, C. A., Nizza, D. T., and Gawrisch, K. (2002) Nuclear Overhauser enhancement spectroscopy cross-relaxation rates and ethanol distribution across membranes, Biophys. J. 82, 1396-1404.

27. Gawrisch, K., and Holte, L. L. (1996) NMR investigations of nonlamellar phase promoters in the lamellar phase state, Chem. Phys. Lipids 81, 105-116.

28. Marsh, D. (1996) Lateral pressure in membranes, Biochim. Biophys. Acta 1286, 183-223.

29. Bezrukov, S. M. (2000) Functional consequences of lipid packing stress, Curr. Opin. Colloid Interface Sci. 5, 237-243.

30. Urban, B. W. (2002) Current assessment of targets and theories of anaesthesia, Br. J. Anaesth. 89, 167-183.

31. Yamakura, T., Lewohl, J. M., and Harris, R. A. (2001) Differential effects of general anesthetics on $\mathrm{G}$ protein-coupled inwardly rectifying and other potassium channels, Anesthesiology 95, 144153.

32. Spencer Yost, C. (1999) Potassium Channels: Basic Aspects, Functional Roles, and Medical Significance, Anesthesiology 90, 1186-1204.

33. Stadnicka, A., Bosnjak, Z. J., Kampine, J. P., and Kwok, W. M. (2000) Modulation of cardiac inward rectifier $\mathrm{K}^{+}$current by halothane and isoflurane, Anesth. Analg. 90, 824-833.

34. Meyer, H. H. (1899) Theorie der Alkoholnarkose, Arch. Exp. Pathol. Pharmakol. 42, 109-118.

35. Overton, E. (1901) Studien uber die Narkose, Zugleich ein Beitrag zur allgemeinen Pharmakologie, Gustav Fischer, Jena, Germany.

36. Dluzewski, A. R., Halsey, M. J., and Simmonds, A. C. (1983) Membrane interactions with general and local anaesthetics: a review of molecular hypotheses of anaesthesia, Mol. Aspects Med. 6, 461-573.

37. Franks, N. P., and Lieb, W. R. (1994) Molecular and cellular mechanisms of general anaesthesia, Nature 367, 607-614.

38. Cantor, R. S. (1997) The lateral pressure profile in membranes: a physical mechanism of general anesthesia, Biochemistry 36 , $2339-2344$.

39. Cantor, R. S. (1998) The lateral pressure profile in membranes: a physical mechanism of general anesthesia, Toxicol. Lett. 100, 451-458.

40. Cantor, R. S. (2001) Breaking the Meyer-Overton rule: predicted effects of varying stiffness and interfacial activity on the intrinsic potency of anesthetics, Biophys. J. 80, 2284-2297.

41. Cantor, R. S. (2003) Receptor desensitization by neurotransmitters in membranes: are neurotransmitters the endogenous anesthetics? Biochemistry 42, 11891-11897.

42. Abraham, M. H., Chadha, H. S., Whiting, G. S., and Mitchell, R. C. (1994) Hydrogen bonding. 32. An analysis of water-octanol and water-alkane partitioning and the DlogP parameter of Seiler, J. Pharm. Sci. 83, 1085-1100.

43. Godden, E. L., Harris, R. A., and Dunwiddie, T. V. (2001) Correlation between molecular volume and effects of $n$-alcohols on human neuronal nicotinic acetylcholine receptors expressed in Xenopus oocytes, J. Pharmacol. Exp. Ther. 296, 716-722.

BI0496079 